\title{
THE MODULI SPACE OF A PUNCTURED SURFACE AND PERTURBATIVE SERIES
}

\author{
R. C. PENNER
}

0. Introduction. Let $F_{g}^{s}$ denote the oriented genus $g$ surface with $s$ punctures, $2 g-2+s>0, s \geq 1$, and choose a distinguished puncture $P$ of $F_{g}^{s}$. Let $\tau_{g}^{s}$ be the Teichmüller space of conformal classes of complete finitearea metrics on $F_{g}^{s}$ (see $[\mathbf{A}]$ ), and let $M C_{g}^{s}$ denote the mapping class group of orientation-preserving diffeomorphisms of $F_{g}^{s}$ (fixing $P$ ) modulo isotopy (see $[B])$. When $g, s$ are understood, we omit their mention. In $\S 1$ and $\S 2$, we report on joint work with D. B. A. Epstein [EP] where new and useful coordinates on $\tau_{g}^{s}$ are given (Theorem 2) and a $M C_{g}^{s}$-equivariant cell decomposition of $\tau_{g}^{s}$ is described (Theorem 3). There is thus an induced cell decomposition of the quotient $\mathcal{M}_{g}^{s}=\tau_{g}^{s} / M C_{g}^{s}$, which is the usual moduli space of $F_{g}^{s}$ in case $s=1$. In $\S 3$, we describe a remarkable connection (see $[\mathbf{P}]$ ) between this cell-decomposition for $s=1$ and a technique from quantum field theory, which allows the computation of certain numerical invariants of $\mathcal{M}_{g}^{s}$ (Corollary 6). Analogues of Theorem 3 have been obtained independently by [BE and H] using different techniques. Furthermore, Corollary 7 is in agreement with some recent work in $[\mathbf{H Z}]$.

Let $M$ denote Minkowskii 3-space with bilinear pairing $\langle\cdot, \cdot\rangle$ of type $(+,+,-)$, and let $L^{+} \subset M$ denote the (open) positive light-cone. The uniformization theorem (see $[\mathbf{A}]$ ) allows us to identify $\tau_{g}^{s}$ with the space of (conjugacy classes of faithful and discrete) representations of $\pi_{1}\left(F_{g}^{s}\right)$ in $S O(2,1)$ (as a Fuchsian group of the first kind in the component of the identity).

I. Coordinates on $\tau$. Suppose $\pi_{1} F=\Gamma \in \tau$, and choose a parabolic transformation $\gamma \in \Gamma$ corresponding to the puncture $P$. $\gamma$ fixes a unique ray in $L^{+}$, and we choose a point $z \in L^{+}$in this ray. If $c$ is a bi-infinite geodesic in $F$ which tends in both directions to $P$ (to be termed simply a geodesic in the sequel), let $\gamma(c) \in \Gamma$ denote the corresponding translation, and define the $\lambda$-length of (the homotopy class of) $c$ to be $\lambda_{\Gamma}(c)=\sqrt{-\langle z, \gamma(c) z\rangle}$. When $\Gamma$ is understood, we denote $\lambda(c)=\lambda_{\Gamma}(c)$. If $h$ is a $\Gamma$-horosphere about $P$ and $c$ is a $\Gamma$-geodesic, then we define $d_{h}(c)$ to be the $\Gamma$-hyperbolic length along $c$ from $h$ back to $h$.

LEMMA 1. If $c_{1}$ and $c_{2}$ are geodesics, then

$$
\lim _{h \rightarrow P} \exp \left\{d_{h}\left(c_{1}\right)-d_{h}\left(c_{2}\right)\right\}=\left[\lambda\left(c_{1}\right) / \lambda\left(c_{2}\right)\right]^{2} .
$$

It follows that $\lambda$-lengths are natural in the sense that if $\varphi \in M C, \Gamma \in \tau$, and $c_{1}, c_{2}$ are geodesics, then $\lambda_{\varphi^{*} \Gamma}\left(c_{1}\right) / \lambda_{\varphi^{*} \Gamma}\left(c_{2}\right)=\lambda_{\Gamma}\left(\varphi^{-1} c_{1}\right) / \lambda_{\Gamma}\left(\varphi^{-1} c_{2}\right)$,

Received by the editors April 29, 1985 and, in revised form, December 18, 1985.

1980 Mathematics Subject Classification (1985 Revision). Primary 14H15, 30F35, 57N05; Secondary 05C30. 
where $\varphi^{*}$ denotes push-forward of conformal type by $\varphi$; moreover, the set of ratios of $\lambda$-lengths for a fixed $\Gamma$ are discrete.

We define an ideal triangulation $\Delta$ of $F$ to be a decomposition of $F$ by geodesics into regions whose doubles are thrice-punctured spheres.

THEOREM 2. $\lambda$-lengths of edges of $\Delta$ give $\mathbf{R}$-analytic projective coordinates on $\tau$. Furthermore, $M C$ acts on these coordinates by polynomials.

For the first part of the theorem, we use the $\lambda$-length data to build an ideal tesselation of the hyperbolic plane and apply Poincaré's Theorem to associate a conformal structure on $F$. For the second part, we claim that the following move on ideal triangulations acts transitively: remove the diagonal $e$ of an ideal quadrilaterial $Q$ of $\Delta$, replacing it by the other diagonal $f$ of $Q$. (This well-known result follows from our Theorem 3 below.) If $(a, c)$, $(b, d)$ are the pairs of opposite edges of $Q$, then one computes in $M$ that $\lambda(e) \lambda(f)=\lambda(a) \lambda(c)+\lambda(b) \lambda(d)$. Since $\lambda$-lengths are natural by Lemma 1 , the theorem follows.

REMARKS. (1) The previous equation is exactly Ptolemy's theorem on side lengths of a Euclidean quadrilateral inscribed in a circle.

(2) The action of $M C$ on $\lambda$-lengths is explicit; computer work has been done.

II. The cell decomposition of $\tau$. Consider now the orbit $\Gamma z$ of $z$ in $L^{+}$. Lemma 1 guarantees that $\Gamma z$ does not accumulate in $L^{+}$(even though the action of $\Gamma$ on $L^{+}$is ergodic). Furthermore, the extremal edges of the (Euclidean) convex hull of $\Gamma z$ can be shown to project to a collection $\Delta(\Gamma)$ of disjoint geodesics in $F$, and regions of $F-\Delta(\Gamma)$ are either simply connected or puncture-parallel; we call such a collection of geodesics in $F$ an ideal cell decomposition of $F$. Let $\nLeftarrow$ denote the poset consisting of all

$$
C(\Delta)=\{\Gamma \in \tau: \Delta(\Gamma)=\Delta\}, \quad \Delta \text { an ideal cell decomposition, }
$$

where $C(\Delta)<C\left(\Delta^{\prime}\right)$ if $\Delta \subset \Delta^{\prime}$.

THEOREM 3. H is an MC-equivariant cell decomposition of $\tau$. Furthermore, $\forall$ extends naturally to a cell decomposition of a natural compactification $\tau$ of $T$ so that cells are finite-sided.

To prove Theorem 3, one first describes $C(\Delta)$ in $\lambda$-length coordinates (with respect to $\Delta$ ) by a system of coupled nonlinear inequalities. Again using naturality of $\lambda$-lengths, we see that $\sharp$ is an $M C$-invariant decomposition of $\tau$, and it remains to check that each $C(\Delta)$ is contractible. We describe a contraction on the set of $\lambda$-lengths parametrizing $C(\Delta)$, which respects the system of inequalities; this step is quite delicate and involves some estimates on side lengths of Euclidean polygons. (See Remark 1 above.) The extension to a compactification is accomplished by adjoining cells corresponding to families of geodesics disjointly embedded in $F$ which are not ideal cell decompositions.

The duals of ideal cell decompositions of $F_{g}^{s}$ are certain spines $G$ in $F_{g}^{s}$, and if $s=1$, then all spines arise. We will also consider the dual spine $\Sigma$ of $\nvdash$ on $T$. Cells of $\Sigma$ are given by (isotopy classes of certain) pairs $(F, G)$ with 
partial ordering given by Whitehead collapses of graphs. $\Sigma$ is contractible, and $M C$ acts on $\Sigma$ with finite isotropy groups.

REMARK. Suppose $G \subset F$ is a spine of $F$ which is dual to a cell decomposition. There is a $\Gamma_{G} \in \tau$ so that the topological symmetry group of the pair $(F, G)$ acts as a group of conformal symmetries of $\Gamma_{G}$. The corresponding matrix groups are explicitly computable and have interesting diophantine properties.

III. Perturbative series computations. A well-known numerical invariant of the moduli space $\mathcal{M}_{g}^{1}$ of $F_{g}^{1}$ is the virtual (or orbifold) Euler characteristic $\chi_{g}=\chi(Z) /\left[M C_{g}^{1}: Z\right]$, where $Z<M C_{g}^{1}$ is finite-index torsion-free and $\chi(Z)$ is the usual Euler characteristic. We will compute $\chi_{g}$ along with a collection of further numerical invariants.

We define a fat graph $G$ to be a planar projection of a graph in $\mathbf{R}^{3}$; each vertex of $G$ is required to be at least tri-valent. A neighborhood of the vertex set inherits an orientation from $\mathbf{R}^{2}$, and we attach orientation-preserving bands along the edges of $G$ to build a pair $(F(G), G)$, where $G$ is a spine of the surface $F(G)$. We define $v_{k}(G)=\#\{k$-valent vertices of $G\}, \lambda(G)=\#\{$ boundary components of $F(G)\}$, and let $\Gamma(G)([G]$, resp.) denote the automorphism group (isomorphism class, resp.) of the oriented pair $(F(G), G)$.

In what follows, we compute

$$
\phi(I, N)=\sum_{[G] \text { with }-2 \chi(G)=I} \frac{(-1)^{\Sigma v_{k}(G)} N^{\lambda(G)}}{\# \Gamma(G)} .
$$

The argument $I$ determines the Euler characteristic of contributing fat graphs, and for each $I, \phi$ is a polynomial in $N$. As a special case, the end of $\S 2$ guarantees that $\chi_{g}$ is the coefficient of $N$ in $\phi(4 g-2, N)$ which can be taken as motivation for our interest in $\phi$.

THEOREM 4. We have the following equality: ${ }^{1}$

$$
\begin{aligned}
\phi(I, N)= & \sum_{\substack{\text { tuples } v_{k} \text { with } \\
\Sigma(k-2) v_{k}=I \\
\text { so that } v_{1}=v_{2}=0}} \frac{(-1)^{\Sigma v_{k}}}{2^{N / 2} \pi^{N^{2} / 2} \prod_{k \geq 1} v_{k} !} \\
& \cdot \int_{M \in H^{N}} \prod_{k \geq 1}\left[\frac{\operatorname{trace} M^{k}}{k}\right]^{v_{k}} \exp \left(-\frac{1}{2} \text { trace } M^{2}\right) d M,
\end{aligned}
$$

where $d M$ is the unitary-invariant product of Lebesgue measures

$$
d M=\left(\prod_{i=1}^{N} d M_{i i}\right) \prod_{1 \leq i<j \leq N} d\left(\operatorname{Re} M_{i j}\right) d\left(\operatorname{Im} M_{i j}\right)
$$

on the $N \times N$ Hermitians $H^{N}$.

The technique of proof is an extension of one known amongst physicists as Feynman diagrams/perturbative series (see [BIZ]) and will not be taken up

\footnotetext{
${ }^{1}$ NOTE ADDED IN PROOF. The right-hand side of this equality bears a striking resemblance to Weil's formula for the total Chern class of a bundle.
} 
here. It is remarkable that this technique so effectively captures the combinatorics of our complex $\Sigma$, at least when $s=1$.

THEOREM 5. We have the following equality.

$$
\phi(2 I, N)=\left.\frac{(-1)^{I}}{I !} \frac{\partial^{I}}{\partial t^{I}}\right|_{t \downarrow 0}\left[\frac{\sqrt{2 \pi t}(e t)^{-t^{-1}}}{\Gamma\left(t^{-1}\right)}\right]^{N} \prod_{p=1}^{N-1}(1-p t)^{N-p} .
$$

To prove this result, one passes from the integral in Theorem 4 to an integral over $\mathbf{R}^{N}$. In so doing, one introduces the factor $\prod_{1 \leq i<j \leq N}\left(x_{i}-x_{j}\right)^{2}$ in the integrand. The integral of such a factor against $\prod_{i=1, N} d \mu\left(x_{i}\right)$ for some measure $d \mu$ on $\mathbf{R}$ can be expressed in terms of the zeroth moment of $d \mu$ and the coefficients of the recursion relation for the orthogonal polynomials of $d \mu$. We construct a generating function $\hat{\phi}(t, N)$ for the quantities $\phi(I, N)$ so that the resulting integral is of this form for some measure $d \mu_{t}$ whose orthogonal polynomials and moments are explicitly computable. Unfortunately, there are convergence problems for $t=0$, and we must truncate the integrals spatially and take limits to pursue this program. Thus, $\{\phi(2 I, N): I \geq 0\}$ arises as the set of coefficients in the asymptotic series at zero of the function in Theorem 5 .

We define $\theta(I, N)$ similarly to $\phi(I, N)$ but summing only over connected fat graphs. Taking logarithmic derivatives and using a variant of Stirling's formula gives our main result on fat graphs.

COROLLARY 6. We have the following equality.

$$
\theta(2 I, N)=(-1)^{I}\left[\frac{-N^{I+2}}{I(I+1)(I+2)}+\sum_{k=1}^{[(I+1) / 2]}\left(\begin{array}{c}
I-1 \\
2 k-2
\end{array}\right) \frac{B_{2 k}}{2 k} \frac{N^{I+2-2 k}}{I+2-2 k}\right],
$$

where $B_{2 k}$ is the $2 k$ th Bernoulli number and [.] denotes integral part.

Since a fat graph with $\lambda=1$ is necessarily connected, we find

COROLlARY 7. The virtual Euler characteristic of moduli space is

$$
\chi_{g}=\text { coefficient of } N \text { in }(\theta(4 g-2, N))=\frac{-B_{2 g}}{2 g}=\varsigma(1-2 g),
$$

where $\varsigma$ is the Riemann zeta function.

Only a small part of the data about fat graphs obtained in Corollary 6 is used in Corollary 7. The remaining information is likely to be related to the action of $M C_{g}^{s}$ on our decomposition of $\bar{\tau}_{g}^{s}$.

\section{REFERENCES}

[A] W. Abikoff, The real-analytic theory of Teichmüller space, Lecture Notes in Math., vol. 820, Springer-Verlag, Berlin and New York, 1980.

[B] J. Birman, Braids, links, and mapping class groups, Ann. of Math. Studies, no. $82,1974$.

[BE] B. Bowditch and D. B. A. Epstein, Triangulations associated with punctured surfaces, preprint (1985). 
[BIZ] D. Bessis, C. Itzykson, and J. B. Zuber, Quantum field theory techniques in graphical enumeration, Adv. Appl. Math. 1 (1980), 109-157.

[EP] D. B. A. Epstein and R. C. Penner, Teichmüller spaces of punctured surfaces, preprint (1984).

[H] J. Harer, The virtual cohomological dimension of the mapping class group of an oriented surface, Invent. Math. (1986).

[HZ] J. Harer and D. Zagier, The Euler characteristic of the moduli space of curves, preprint (1985).

[P] R. C. Penner, Perturbative series and moduli space, preprint (1985).

DEPARTMENT OF MATHEMATICS, UNIVERSITY OF SOUTHERN CALIFORNIA, LOS ANGELES, CALIFORNIA 90089 